\title{
New Models of Dark Energy Stars with Charge Distributions
}

\author{
Manuel Malaver ${ }^{1,2}$, María Esculpi ${ }^{3}$, Megandhren Govender ${ }^{4}$ \\ ${ }^{1}$ Bijective Physics Institute, Idrija, Slovenia \\ ${ }^{2}$ Department of Basic Sciences, Maritime University of the Caribbean, Catia la Mar, Venezuela \\ ${ }^{3}$ Department of Applied Physics, Central University of Venezuela, Faculty of Engineering, Caracas, Venezuela \\ ${ }^{4}$ Department of Mathematics, Statistical and Physics, Durban University of Technology, Durban, South Africa
}

Email address:

mmf.ume@gmail.com (M. Malaver),mariaesculpiw@gmail.com (M. Esculpi), govenderm43@ukzn.ac.za (M. Govender)

\section{To cite this article:}

Manuel Malaver, María Esculpi, Megandhren Govende. New Models of Dark Energy Stars with Charge Distributions. International Journal of Astrophysics and Space Science. Vol. 7, No. 2, 2019, pp. 18-23. doi: 10.11648/j.ijass.20190702.12

Received: July 15, 2019; Accepted: August 19, 2019; Published: September 2, 2019

\begin{abstract}
In this paper, we have obtained a relativistic and spherically symmetric stellar configuration that describes an anisotropic fluid with a charge distribution that represents a potential model for a dark energy star and we specify particular forms in the gravitational potential and the electric field intensity which allows solve the Einstein-Maxwell field equations. The reason for proposing this model originates from the evidence that recent observational findings suggest that the universe has an accelerated cosmic expansion and the model of dark energy star is one of the most reasonable explanations of this phenomena. The field equations are integrated analytical and new stellar configurations are obtained are analyzed. For each these solutions we found that the radial pressure, the anisotropy factor, energy density, metric coefficients, mass function, charge density are regular and well behaved in the stellar interior. With the new solutions can be developed models of dark energy stars physically acceptable where the causality condition is not satisfied or the strong energy condition is violated. This model has a great application in the study of the fundamental theories of physics and cosmology. Several independent observations indicate that the greater part of the total energy density of the universe is in the form of dark energy and the rest in the form of nonbaryonic cold dark matter particles, but which have never been detected.
\end{abstract}

Keywords: Stellar Configuration, Gravitational Potential, Charge Distribution, Anisotropic Fluid, Accelerated Cosmic Expansion, Einstein-Maxwell Field Equations

\section{Introduction}

Recent astrophysical observations have confirmed that the Universe shows an accelerated cosmic expansion [1]. Evidence of this expansion has been shown independently from measurements of supernovae of type Ia and from microwave background radiation [2]. It is proposed that this cosmological behavior is caused by a hypothetical dark energy, a cosmic fluid parameterized by an equation of state $\omega=p / \rho<-1 / 3$ where $\mathrm{p}$ is the spatially homogeneous pressure and $\rho$ the dark energy density [1-4]. The dark energy possesses a strong negative pressure that can help to explain the acceleration of the universe in expansion [4]. The range for which $\omega<-1$ has been denoted phantom energy and possesses peculiar properties, such as negative temperatures and the energy density increases to infinity in a finite time, resulting in a big rip [2-4]. It also provides a natural scenario for the existence of exotic geometries s uch as wormholes [5-7].

In the construction of the first theoretical models of relativistic objects are important the works of Schwarzschild [8], Tolman [9], Oppenheimer and Volkoff [10]. Schwarzschild [8] found analytical solutions that allowed describing a star with uniform density, Tolman [9] developed a method to find solutions of static spheres of fluid and Oppenheimer and Volkoff [10] used Tolman's solutions to study the gravitational balance of neutron stars. It is important to mention Chandrasekhar's contributions [11] in the model production of white dwarfs in presence of relativistic effects and the works of Baade and Zwicky [12] who propose the concept of neutron stars and identify a 
astronomic dense objects known as supernovas.

The notion of dark energy is that of a homogeneously distributed cosmic fluid and when extended to inhomogeneous spherically symmetric spacetimes, the pressure appearing in the equation of state is now a negative radial pressure, and the tangential pressure is then determined via the field equations [2,3]. The generalization of the gravastars picture with the inclusion of an interior solution governed by the equation of state $p=\omega \rho$ with $\omega<-1 / 3$, will be denoted by dark energy star in agreement with Chapline [13]. Lobo [3] explored several configurations, by imposing specific choices for the mass function and studied the dynamical stability of these models by applying the general stability formalism developed by Lobo and Crawford [14]. Chan et al. [15] propose that the mass function is a natural consequence of the Einstein's field equations and considered a core with a homogeneous energy density, described by the Lobo's first solution [3]. Malaver and Esculpi [16] presented a new model of dark energy star by imposing specific choice for the mass function which correspond an increase in energy density inside of the star. Bibi et al. [4] obtained a new class of solutions of the Einstein-Maxwell field equations which represents a model for dark energy stars with the equation of state $p_{r}=-\rho$. According Chan et al. [15] the denomination dark energy is applied to fluids which violate only the strong energy condition given by $\rho+p_{r}+2 p t \geq 0$ where $\rho$ is the energy density, $p_{r}$ and $p_{t}$ are the radial pressure and tangential pressure, respectively.

In the description of the behavior of relativistic gravitating matter is important include the presence of anisotropy in the pressure [17-29] and is defined as $p_{t}-p_{r}$ where $p_{t}$ is the tangential pressure. Bowers and Liang [30] extensively discuss the effect of anisotropy in general relativity. The existence of anisotropy within a star can be explained by the presence of a solid core, phase transitions, a type III super fluid, a pion condensation [31] or another physical phenomenon as the presence of an electrical field [32]. Many researchers have used a great variety of mathematical techniques to try to obtain solutions of the Einstein-Maxwell field equations since it has been demonstrated Komathiraj and Maharaj [33], Thirukkanesh and Maharaj [34], Maharaj et al. [35], Thirukkanesh and Ragel [36, 37], Feroze and Siddiqui [38, 39], Sunzu et al. [40], Pant et al. [41] and Malaver [42-45].

Following Bibi et al. [4], in this paper we obtain a new class of solutions of the fields equations that represents a model of dark energy stars whose equation of state is $p_{r}=\omega \rho$ using a specify forms for the gravitational potential and the electric field intensity. We assume that the denomination dark energy is applied to fluids which violate the strong energy condition [15]. This article is organized as follows, in Section 2, we present Einstein's field equations. In Section 3, we make a particular choice of gravitational potential $Z(x)$ that allows solving the field equations and we have obtained new models for dark energy stars consistent alone of dark matter. In Section 4, a physical analysis of the new solutions is performed. Finally in Section 5, we conclude.

\section{Einstein-Maxwell Field Equations}

We consider a spherically symmetric, static and homogeneous spacetime. In Schwarzschild coordinates the metric is given by

$$
d s^{2}=-e^{2 v(r)} d t^{2}+e^{2 \lambda(r)} d r^{2}+r^{2}\left(d \theta^{2}+\sin ^{2} \theta d \varphi^{2}\right)
$$

where $v(r)$ and $\lambda(r)$ are two arbitrary functions.

The Einstein field equations for the charged anisotropic matter are given by

$$
\begin{gathered}
\frac{1}{r^{2}}\left(1-e^{-2 \lambda}\right)+\frac{2 \lambda^{\prime}}{r} e^{-2 \lambda}=\rho+\frac{1}{2} E^{2} \\
-\frac{1}{r^{2}}\left(1-e^{-2 \lambda}\right)+\frac{2 v^{\prime}}{r} e^{-2 \lambda}=p_{r}-\frac{1}{2} E^{2} \\
e^{-2 \lambda}\left(v^{\prime \prime}+v^{\prime 2}+\frac{v^{\prime}}{r}-v^{\prime} \lambda^{\prime}-\frac{\lambda^{\prime}}{r}\right)=p_{t}+\frac{1}{2} E^{2} \\
\sigma=\frac{1}{r^{2}} e^{-\lambda}\left(r^{2} E\right)^{\prime}
\end{gathered}
$$

where $\rho$ is the energy density, $p_{r}$ is the radial pressure, $E$ is electric field intensity, $p_{t}$ is the tangential pressure and primes denote differentiations with respect to $r$. Using the transformations, $x=c r^{2}, Z(x)=e^{-2 \lambda(r)}$ and $A^{2} y^{2}(x)=e^{2 v(r)}$ with arbitrary constants $A$ and $c>0$, suggested by Durgapal and Bannerji [46], the Einstein field equations can be written as

$$
\begin{gathered}
\frac{1-Z}{x}-2 \dot{Z}=\frac{\rho}{c}+\frac{E^{2}}{2 c} \\
4 Z \frac{\dot{y}}{y}-\frac{1-Z}{x}=\frac{p_{r}}{c}-\frac{E^{2}}{2 c} \\
4 x Z \frac{\ddot{y}}{y}+(4 Z+2 \times \dot{Z}) \frac{\dot{y}}{y}+\dot{Z}=\frac{p_{t}}{c}+\frac{E^{2}}{2 c} \\
\frac{\Delta}{c}=4 x Z \frac{\ddot{y}}{y}+\dot{Z}\left(1+2 x \frac{\dot{y}}{y}\right)+\frac{1-Z}{x}-\frac{E^{2}}{c} \\
\sigma^{2}=\frac{4 c Z}{x}(x \dot{E}+E)^{2}
\end{gathered}
$$

$\sigma$ is the charge density, $\Delta=p_{t}-p_{r}$ is the anisotropy factor and dots denote differentiation with respect to $\mathrm{x}$. With the transformations of [46], the mass within a radius $r$ of the sphere takes the form

$$
M(x)=\frac{1}{4 c^{3 / 2}} \int_{0}^{x} \sqrt{x}\left(\rho^{*}+E^{2}\right) d x
$$


where

$$
\rho^{*}=\left(\frac{1-Z}{x}-2 \dot{Z}\right) c
$$

The interior metric (1) with the charged matter distribution should match the exterior spacetime described by the Reissner-Nordstrom metric:

$$
d s^{2}=-\left(1-\frac{2 M}{r}+\frac{Q^{2}}{r^{2}}\right) d t^{2}+\left(1-\frac{2 M}{r}+\frac{Q^{2}}{r^{2}}\right) d r^{2}+r^{2}\left(d \theta^{2}+\sin ^{2} \theta d \varphi^{2}\right)
$$

where the total mass and the total charge of the star are denoted by $\mathrm{M}$ and $\mathrm{q} 2$, respectively. The junction conditions at the stellar surface are obtained by matching the first and the second fundamental forms for the interior metric (1) and the exterior metric (13).

In this paper, we assume the following equation of state

$$
p_{r}=\omega \rho
$$

where $\omega$ is the dark energy parameter.

\section{The New Models for Dark Energy Stars}

In order to solve the Einstein field equations, we have chosen specific forms for the gravitational potential $\mathrm{Z}$ and the electrical field intensity E. Following MafaTakisa and Maharaj [47] and Bibi et al. [4] we have taken the forms

$$
Z(x)=\frac{1+b x}{1+a x}
$$

$$
E^{2}=\frac{K a x(5+a x)}{(1+a x)^{3}}
$$

The potential is regular at the origin and well behaved in the interior of the sphere. Substituting (15) and (16) in (6) we obtain

$\rho=\frac{\left(2 c a^{3}-2 c b a^{2}-K a^{2}\right) x^{2}+\left(8 c a^{2}-8 c a b-5 K a\right) x+6 c(a-b)}{2(1+a x)^{3}}$

Replacing (17) in (14), we have for the radial pressure

$p_{r}=\omega\left[\frac{\left(2 c a^{3}-2 c b a^{2}-K a^{2}\right) x^{2}+\left(8 c a^{2}-8 c a b-5 K a\right) x+6 c(a-b)}{2(1+a x)^{3}}\right]$

Supposing continuity in the first and second fundamental form, if $p_{r}(r=R)=0$ we have

$$
a^{3}-a^{2}(b+K-4)-a(4 b+3)-\frac{5}{2} K-3 b=0
$$

Using (16) and (17) in (12), the expression of the mass function is

$$
M(x)=x^{3 / 2} \frac{\left[2 c(a-b)+\left(2 a^{2} c-2 a b c-K a\right) x\right]}{c^{3 / 2}(1+a x)^{2}}
$$

And for the charge density

$$
\sigma^{2}=\frac{K c a(1+b x)\left(a^{2} x^{2}+4 a x+15\right)^{2}}{(1+a x)^{6}(5+a x)}
$$

With (14), (15) and (16), the eq. (7) becomes

$$
\frac{4(1+b x)}{(1+a x)} \frac{\dot{y}}{y}=\frac{(a-b)}{(1+a x)}+\frac{K a x(5+a x)}{2 c(1+a x)^{3}}-\omega\left[\frac{\left(2 c a^{3}-2 c a^{2} b-K a^{2}\right) x^{2}+\left(8 c a^{2}-8 c a b-5 K a\right) x+6 c(a-b)}{2 c(1+a x)^{3}}\right]
$$

Integrating (22), we obtain

where

$$
\begin{aligned}
y(x)=c_{1}(1+a x)^{B}(1+b x)^{C} e^{-\frac{D}{2(1+a x)}} \quad B=\frac{\left(-3 K a-3 \omega K a-8 \omega c a b+4 \omega b^{2} c+4 \omega a^{2} c-\omega K b-K b\right)}{8(a-b)^{2} c} & (23) \\
C & =\frac{\left(\begin{array}{l}
-K a^{2}-10 \omega a^{2} b c+2 \omega a^{3} c-\omega K a^{2}-6 a^{2} b c+5 K a b+5 \omega K a b-2 b^{3} c-6 \omega b^{3} c \\
+6 a b^{2} c+14 \omega a b^{2} c+2 a^{3} c
\end{array}\right)}{8(a-b)^{2} b c} \\
D & =\frac{(1+\omega) K}{(a-b) c}
\end{aligned}
$$

The metric functions can be written as

$$
e^{2 \lambda(r)}=\frac{1+a x}{1+b x}
$$

as the metric is continuous, in $\mathrm{r}=\mathrm{R}$ we have

$$
c_{1}^{2}=\frac{\left(1-\frac{2 M}{R}+\frac{Q^{2}}{R^{2}}\right)}{A^{2}(1+a)^{B}(1+b)^{C} e^{D / 1+a}}
$$


The anisotropy factor $\Delta$ is given by for

$$
\begin{aligned}
\Delta= & \frac{4 x c(1+b x)}{(1+a x)}\left[\frac{B^{2} a^{2}-B a^{2}}{(1+a x)^{2}}+\frac{2 a C b(B+D)}{(1+a x)(1+b x)}+\frac{\left(C^{2}-C\right) b^{2}}{(1+b x)^{2}}+\frac{B a^{2} D-a^{2} D}{(1+a x)^{3}}+\frac{D^{2} a^{2}}{4(1+a x)^{4}}\right] \\
& +\frac{(b-a) c}{(1+a x)^{2}}\left[1+2 x\left(\frac{B a}{1+a x}+\frac{C b}{1+b x}+\frac{a D}{4(1+a x)^{2}}\right)\right] \\
& +\frac{(a .-b) c}{(1+a x)}-\frac{K a c x(5+a x)}{(1+a x)^{3}}
\end{aligned}
$$

and the metric for this model is

$$
d s^{2}=-A^{2} c_{1}^{2}\left(1+a c r^{2}\right)^{2 B}\left(1+b c r^{2}\right)^{2 C} e^{-\frac{D}{\left(1+a c r^{2}\right)}} d t^{2}+\left(\frac{1+a c r^{2}}{1+b c r^{2}}\right) d r^{2}+r^{2}\left(d \theta^{2}+\sin ^{2} \theta d \varphi^{2}\right)
$$

The figures $1,2,3,4,5,6$ represent the graphs of $\rho, \sigma^{2}$, $\mathrm{M}(\mathrm{x})$, pr, anisotropy $\Delta$, strong energy condition respectively for different values of $\omega$ in the new obtained models with $\mathrm{a}=0.0175, \mathrm{~b}=0.00329$ and a stellar radius of $\mathrm{r}=1 \mathrm{Km}$. The values of the constants are $\mathrm{c}=\mathrm{K}=1$.

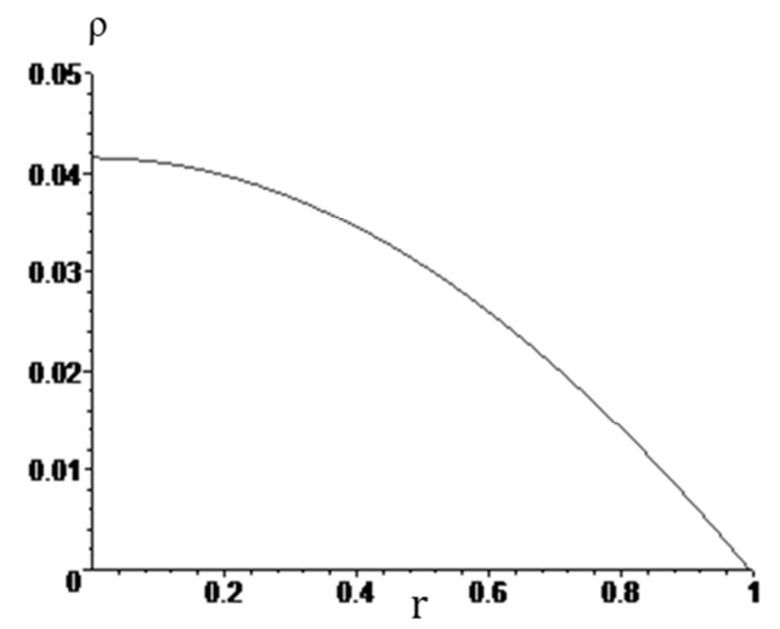

Figure 1. Energy density vs radial coordinate.

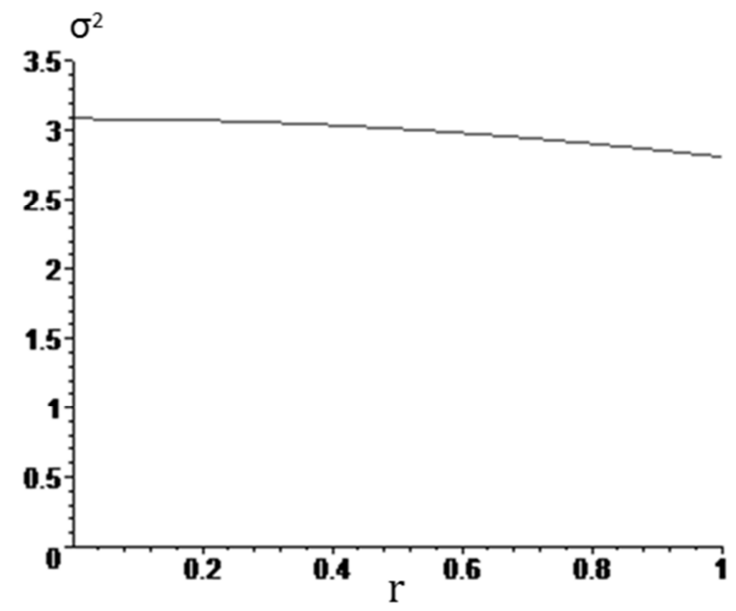

Figure 2. Charge density vs radial coordinate.

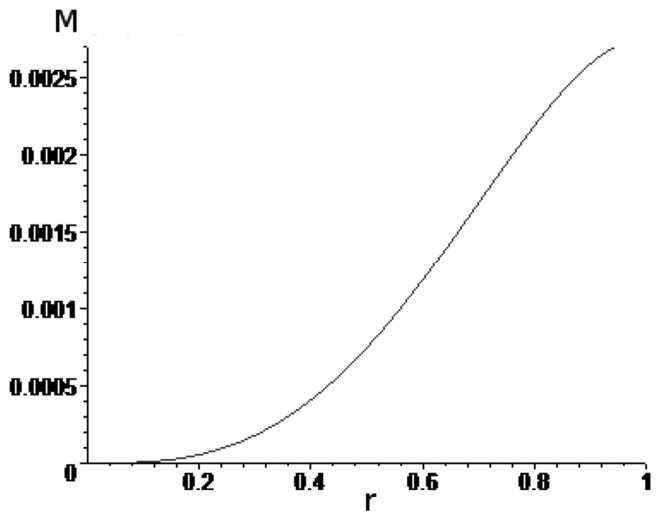

Figure 3. Mass function vs radial coordinate.

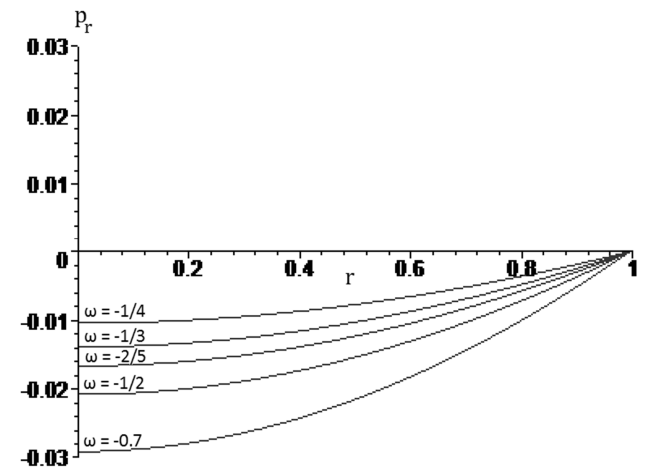

Figure 4. Radial pressure for different values of $\omega$ vs radial coordinate.

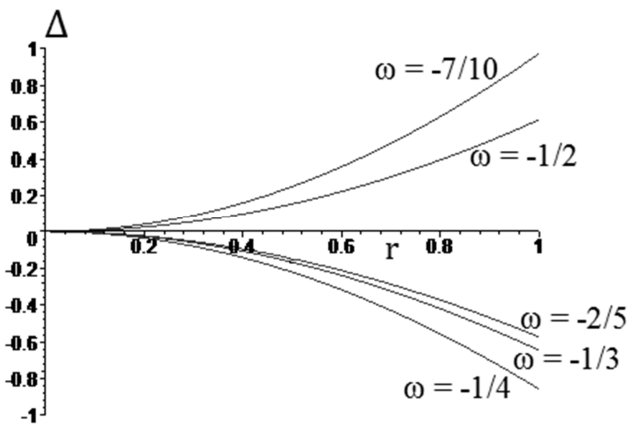

Figure 5. Anisotropy $\Delta$ for different values of $\omega$ vs radial coordinate. 


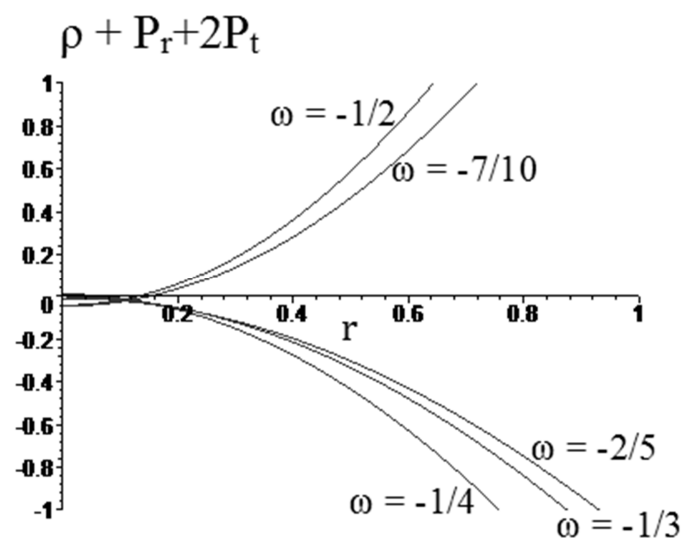

Figure 6. The strong energy condition for different values of $\omega$ vs radial coordinate.

\section{Physical Features of the New Models}

In order for a solution to be physically acceptable and viable must satisfy the following physical properties $[4,15$, 37]:

(i) The energy density must be well defined, must be positive and a decreasing function of the radial pressure

(ii) The radial pressure must decrease as the radius increases and it must vanish at the surface of the sphere but for negative pressure this condition is not satisfied.

(iii) Regularity of the gravitational potentials in the origin.

(iv) For anisotropic solutions, the radial and the tangential pressure are equal to zero at the centre $r=0$

(v) The consideration of dark energy is applicable only to fluids that violate the strong energy condition.

The new obtained models constitute another new family of solutions of Maxwell-Einstein's field equations with anisotropy. The metric functions $\mathrm{e}^{2 \lambda}$ and $\mathrm{e}^{2 v c a n}$ be expressed in terms of elementary functions and the energy density, charge density and mass function also are represented analytical. In $\mathrm{r}=0 \mathrm{we} \quad$ have $\quad \mathrm{e}^{2 \lambda(0)}=1, \quad \mathrm{e}^{2 \mathrm{v}(0)}=A^{2} c_{1}^{2} e^{-C} \quad$ and $\left(e^{2 \lambda(r)}\right)_{r=0}^{\prime}=\left(e^{2 v(r)}\right)_{r=0}^{\prime}=0$. This shows that the gravitational potential is regular at the origin. In the center $=0, \rho=3 c(a-b)$ and $p_{r}=3 c \omega(a-b)$. In these models, the mass function is strictly increasing function, continuous and finite and the charge density behaves well inside of the star and no present singularities at the centre.

In figure 1 , the energy density is finite and decreasing with the radial coordinate. In figure 2 , the charge density is nonsingular at the origin, non-negative and decreases. In figure 3, the mass function is strictly increasing function, continuous and $\mathrm{M}(\mathrm{x})=0$ at $\mathrm{r}=0$. In figure 4 , that represents the variation of the radial pressure for different values of $\omega$, we observe the decrease of $p_{r}$ when it diminishes $\omega$. In figure 5, the anisotropy factor $\Delta$ increases when it diminishes the dark energy parameter $\omega$. In figure 6 it is observed as the strong energy condition is violated for values of $\omega \geq-0.4$ what means that in these new models the consideration of dark energy is valid for this cases.

\section{Conclusions}

We have found a new family of solutions to the EinsteinMaxwell system considering a particular form of the gravitacional potential $\mathrm{Z}(\mathrm{x})$ and the electric field intensity with a linear equation of state that represents a model of dark energy star where $\omega=p / \rho \leq-1 / 3$. The radial, energy density, mass function, anisotropy factor and the metric functions are regular and behaves well inside the stellar interior. The charge density is non-singular at the centre. In this model, the condition of dark energy star is applied only to the cases $\omega \geq$ 0.4 because for these values of $\omega$ is not satisfied the strong energy condition. The new obtained solutions satisfy all the requirements for a compact negative energy stellar object and may be used to model relativistic configurations in different astrophysical scenes.

The main reason for this research arises from that recent observations have verified an accelerated expansion of the Universe and the dark energy is a probable candidate. Evidence of this expansion has been shown independently from measurements of supernovae of type Ia and of the microwave background radiation [2]. This dark energy consists of an cosmic fluid governed by an equation of state $p=\omega \rho$ where the strong energy condition is violated [15]. Following Lobo [3] in this work the limits for the parameter $\omega$ are chosen in a way that $\omega \leq-1 / 3$. The range for which $\omega$ $<-1$ has been denoted phantom energy and provides a natural scenario for the existence of exotic geometries such as traversable wormholes that could be used in interstellar travel with the consequent causality violations $[6,7]$. The dark energy stars could have their origin in a density fluctuation in the cosmological background resulting from the nucleation of a dark energy star through a density perturbation [3].

\section{References}

[1] Sushkov. S. (2005). Wormholes supported by a phantom energy. Phys. Rev. D71, 043520.

[2] Lobo, F. S. N. (2005). Stability of phantom wormholes. Phys. Rev. D71, 124022.

[3] Lobo, F. S. N. (2006). Stable dark energy stars. Class. Quant. Grav. 23, 1525-1541.

[4] Bibi, R., Feroze, T. and Siddiqui, A. (2016). Solution of the Einstein-Maxwell Equations with Anisotropic Negative Pressure as a Potential Model of a Dark Energy Star. Canadian Journal of Physics, 94 (8), 758-762.

[5] Malaver, M. (2013). Black Holes, Wormholes and Dark Energy Stars in General Relativity. Lambert Academic Publishing, Berlin. ISBN: 978-3-659-34784-9.

[6] Morris, M. S. and Thorne, K. S. (1988). Wormholes in spacetime and their use for interstellar travel: A tool for teaching general relativity Am. J. Phys. 56, 395-412.

[7] Visser, M. (1995). Lorentzian wormholes: From Einstein to Hawking. AIP Press, New York. 
[8] Schwarzschild, K. (1916). Uber das Gravitationsfeld einer Kugel aus inkompressibler Flussigkeit nach der Einsteinschen Theorie. Math. Phys. Tech, 424-434.

[9] Tolman, R. C. (1939). Static Solutions of Einstein's Field Equations for Spheres of Fluid. Phys. Rev., 55 (4), 364-373.

[10] Oppenheimer, J. R. and Volkoff, G. (1939). On Massive Neutron Cores. Phys. Rev., 55 (4), 374-381.

[11] Astrophys. J, 74, 81-82. The Maximum Mass of Ideal White Dwarfs Chandrasekhar, S. (1931)

[12] Baade, W. and Zwicky, F. (1934). On Super-novae. Proc. Nat. Acad. Sci. U. S 20 (5), 254-259.

[13] Chapline, G. (2004). Dark Energy Stars. Proceedings of the Texas Conference on Relativistic Astrophysics, 12-17 Stanford.

[14] Lobo, F. S. N. and Crawford, P. (2005). Stability analysis of dynamic thin shells. Class. Quant. Grav, 22, 4869-4886.

[15] Chan, R., da Silva, M. A. F. and Villas da Rocha, J. F. (2009). On Anisotropic Dark Energy. Mod. Phys. Lett A24, 1137-1146.

[16] Malaver, M. and Esculpi, M. (2013). A Theoretical Model of Stable Dark Energy Stars. IJRRAS, 14 (1), 26-39.

[17] Cosenza, M., Herrera, L., Esculpi, M. and Witten, L. (1981). Some Models of Anisotropic Spheres in General Relativity, $J$. Math. Phys, 22 (1), 118.

[18] Gokhroo, M. K. and Mehra, A. L. (1994). Anisotropic Spheres with Variable Energy Density in General Relativity. Gen. Relat. Grav, 26 (1), 75 -84.

[19] Esculpi, M., Malaver, M. and Aloma, E. (2007). A Comparative Analysis of the Adiabatic Stability of Anisotropic Spherically Symmetric solutions in General Relativity. Gen. Relat. Grav, 39 (5), 633-652.

[20] Malaver, M. (2018). Generalized Nonsingular Model for Compact Stars Electrically Charged. World Scientific News, $92(2), 327-339$.

[21] Malaver, M. (2018). Some new models of anisotropic compact stars with quadratic equation of state. World Scientific News, 109, 180-194.

[22] Chan R., Herrera L. and Santos N. O. (1992). Dynamical instability in the collapse of anisotropic matter. Class. Quantum Grav, 9 (10), L133.

[23] Malaver, M. (2017). New Mathematical Models of Compact Stars with Charge Distributions. International Journal of Systems Science and Applied Mathematics, 2 (5), 93-98.

[24] Cosenza M., Herrera L., Esculpi M. and Witten L. (1982). Evolution of radiating anisotropic spheres in general relativity. Phys. Rev. D, 25 (10), 2527-2535.

[25] Herrera L. (1992). Cracking of self-gravitating compact objects. Phys. Lett. A, 165, 206-210.

[26] Herrera L. and Ponce de Leon J. (1985). Perfect fluid spheres admitting a one-parameter group of conformal motions. $J$. Math. Phys, 26, 778.

[27] Herrera L. and Nunez L. (1989). Modeling 'hydrodynamic phase transitions' in a radiating spherically symmetric distribution of matter. The Astrophysical Journal, 339 (1), 339-353.
[28] Herrera L., Ruggeri G. J. and Witten L. (1979). Adiabatic Contraction of Anisotropic Spheres in General Relativity. The Astrophysical Journal, 234, 1094-1099.

[29] Herrera L., Jimenez L., Leal L., Ponce de Leon J., Esculpi M and Galina V. (1984). Anisotropic fluids and conformal motions in general relativity. J. Math. Phys, 25, 3274.

[30] Bowers, R. L. and Liang, E. P. T. (1974). Anisotropic Spheres in General Relativity, The Astrophysical Journal, 188, 657-665.

[31] Sokolov. A. I. (1980). Phase transitions in a superfluid neutron liquid. Sov. Phys. JETP, 52 (4), 575-576.

[32] Usov, V. V. (2004). Electric fields at the quark surface of strange stars in the color- flavor locked phase. Phys. Rev. D, $70(6), 067301$.

[33] Komathiraj, K. and Maharaj, S. D. (2008). Classes of exact Einstein-Maxwell solutions, Gen. Rel. Grav. 39 (12), 2079-2093.

[34] Thirukkanesh, S. and Maharaj, S. D. (2008). Charged anisotropic matter with a linear equation of state. Class. Quantum Gravity, 25 (23), 235001.

[35] Maharaj, S. D., Sunzu, J. M. and Ray, S. (2014). Some simple models for quark stars. Eur. Phys. J. Plus, 129, 3.

[36] Thirukkanesh, S. and Ragel, F. C. (2013). A class of exact strange quark star model. PRAMANA-Journal of physics, 81 (2), 275-286.

[37] Thirukkanesh, S. and Ragel, F. C. (2012). Exact anisotropic sphere with polytropic equation of state. PRAMANA-Journal of physics, 78 (5), 687-696.

[38] Feroze, T. and Siddiqui, A. (2011). Charged anisotropic matter with quadratic equation of state. Gen. Rel. Grav, 43, 1025-1035.

[39] Feroze, T. and Siddiqui, A. (2014). Some Exact Solutions of the Einstein-Maxwell Equations with a Quadratic Equation of State. Journal of the Korean Physical Society, 65 (6), 944-947.

[40] Sunzu, J. M, Maharaj, S. D., Ray, S. (2014). Quark star model with charged anisotropic matter. Astrophysics. Space. Sci, 354, 517-524.

[41] Pant, N., Pradhan, N., Malaver, M. (2015). Anisotropic fluid star model in isotropic coordinates. International Journal of Astrophysics and Space Science. Special Issue: Compact Objects in General Relativity. 3 (1), 1-5.

[42] Malaver, M. (2014). Strange Quark Star Model with Quadratic Equation of State. Frontiers of Mathematics and Its Applications, 1 (1), 9-15.

[43] Malaver, M. (2018). Charged anisotropic models in a modified Tolman IV spacetime. World Scientific News, 101, 31-43.

[44] Malaver, M. (2018). Charged stellar model with a prescribed form of metric function $\mathrm{y}(\mathrm{x})$ in a Tolman VII spacetime. World Scientific News, 108, 41-52.

[45] Malaver, M. (2016). Classes of relativistic stars with quadratic equation of state. World Scientific News, 57, 70-80.

[46] Durgapal, M. C., Bannerji, R. (1983). New analytical stellar model in general relativity. Phys. Rev. D27, 328-331.

[47] Takisa, P. M., Maharaj, S. D. (2013). Some charged polytropic models. Gen. Rel. Grav, 45, 1951-1969. 